\title{
Brain Medical Image Retrieval Using Non-Negative Matrix Factorization and Canny Edge Detection
}

\author{
Ali Akbar Lubis ${ }^{1}$ and Suharjito ${ }^{2}$ \\ ${ }^{1}$ Faculty of Information Technology, Mikroskil, Medan, Indonesia \\ ${ }^{2}$ Magister of Information Technology, Bina Nusantara University, Jakarta, \\ Indonesia \\ ali_akbarlubis@yahoo.com,harjito@yahoo.com
}

\begin{abstract}
Disease conditions in the human brain can be detected by using medical image analysis. Content Based Image Retrieval of medical images can be used as an alternative to recognize the medical images of the human brain. In CBIR, feature extraction and recognition methods was an important feature because of medical image was different from the general image. In this study medical images that contain clinical information will be used as feature extraction using a canny edge detection and recognition features using non-negative matrix Factorization (NNMF). The purpose of this paper was to describe the use of canny edge detection and NNMF in CBIR. So CBIR could provide information on brain diseases and abnormalities. The results showed that the detection of disease in the human brain can be done by using both methods with good results if done preprocessing using histogram equalization.
\end{abstract}

Keywords: Retrieval Image, CBIR, Medical Image, Non-Negative Matrix Factorization

\section{Introduction}

Brain is an organ that often suffered with abnormalities. Some disorders that can occur in the brain is alzheimer or brain cancer (glioma). To make it easier to identify abnormalities in brain, Content Based Image Retrieval (CBIR) can be used as a tool. CBIR system uses visual information which extracted from image to retrieve most similar image which the query image. This system doesn't use the diagnostic information for image retrieval but recognize a specific pattern.

Retrieval process of medical image have differences with other digital images. Medical images have similar visual features among one and the others, therefore, to compare between two similar medical images would be very difficult and retrieval performance will be reduced. To resolve this problem, the most important thing in developing a CBIR system for medical images is how to determine the effective image feature.

To improve performance of images feature extraction, effective methods of representative features are required to extract features from medical images [1]. Some method for feature extraction can be uses in medical image retrieval are canny edge detection [2], exact legendre moments [3], and wavelet transformation [4].

Identify existing features used Non-negative Matrix Factorization (NNMF). NNMF has been implemented into various applications such as face recognition [5], image classification [6], facial expression recognition [7], and face detection [8]. NNMF is a method for describing data into two factors with nonnegative entries [9]. NNMF can reduce the dimension of image [10]. NNMF have good accuracy when using all the information in the image as feature. Results of NNMF reconstruction produce quality images that are similar to the original image. 


\section{Related Work}

Medical image contain feature-richer than general image. This is main between image retrieval systems of medical images and non-medical images [11]. These feature are extracted by using canny edge detection. The canny edge detection algorithm is known to many as the optimal edge detector [12]. The canny edge detector smoothes the image to eliminate noise. It then finds the image gradient to highlight regions with high spatial derivatives. The algorithm then tracks along these regions and suppresses any pixel that is not at the maximum (non-maximum suppression). The gradient array is now further reduced by hysteresis. Hysteresis is used to track along the remaining pixels that have not been suppressed. Hysteresis uses two thresholds and if the magnitude is below the first threshold, it is set to zero (made a non-edge). If the magnitude is above the high threshold, it is made an edge [13].

After feature extraction with canny edge detection, Dimension reduction process will be done by using NNMF method. Non-Negative Matrix Factorization (NMF) is a linear dimension reduction technique which has a non-negative constraints. These constraints produced a partial based representation because these constraits only allow addition, not reduction or combination of both [14]. Non-Negative Matrix Factorization produced a non-negative matrix called $\mathrm{X}$. X matrix contains the data that will be used for feature recognize. Then there are 3 matrix is used to make image factorization with NNMF method.

$$
X_{i \mu} \approx(W H)_{i \mu}=\sum_{a=1}^{r} W_{i a} H_{a \mu}
$$

For instance, $\mathrm{W}$ is a $\mathrm{n} * \mathrm{r}$ matrix and $\mathrm{H}$ is a $\mathrm{r} * \mathrm{~m}$ matrix, so if $\mathrm{W}$ is multiplied by the $\mathrm{H}$ matrix, it will generate $\mathrm{X}$ which is a $\mathrm{n} \mathrm{x}$ m matrix. Each column in $\mathrm{X}$ matrix containing the non-negative pixel value of each row. W matrix is referred as the base image, while the matrix $\mathrm{H}$ is referred as encoding and each column in $\mathrm{H}$ matrix associated with the existing image data in $\mathrm{X}$ matrix. The value of $\mathrm{r}$ value in $\mathrm{W}$ and $\mathrm{H}$ matrix usually obtained from the results of the experiment with conditions $(n+m) * r<n m$, and the results of the $\mathrm{W}^{*} \mathrm{H}$ can be considered as a form of data compression of $\mathrm{X}$. Each value in matrix must be positive. Because of non-negative constraint made on decomposition process, reconstruction of images is formed with the addition of the image-based composition.

Based on matrix factorization of formula NNMF, it is necessary to find $\mathrm{W}$ and $\mathrm{H}$ matrix from $\mathrm{X}$ matrix, which $\mathrm{W}$ and $\mathrm{H}$ matrix contains a database of images. Formula to find the matrix $\mathrm{W}$ and $\mathrm{H}$ are:

$$
\begin{aligned}
& W_{i a} \leftarrow W_{i a} \sum_{\mu} \frac{X_{i \mu}}{(W H)_{i \mu}} H_{a \mu} \\
& W_{i a} \leftarrow \sum_{j} \frac{W_{i a}}{W_{j a}} \\
& H_{a \mu} \leftarrow H_{a \mu} \sum_{\mu} W_{i a} \frac{X_{i \mu}}{(W H)_{i \mu}}
\end{aligned}
$$

The initial process in NNMF is creating a random non negative $\mathrm{W}$ and $\mathrm{H}$ matrix. And then using the above formula, the most optimal matrix $\mathrm{W}$ and $\mathrm{H}$ will determine. $\mathrm{W}$ and $\mathrm{H}$ will be used for multiplication process to find matrix that closet to $\mathrm{X}$ [15].

\section{Methodology}

Medical image retrieval process consists of two main processes which are training and retrieval process. The methodology used in retrieval process can be seen in Figure 1. 


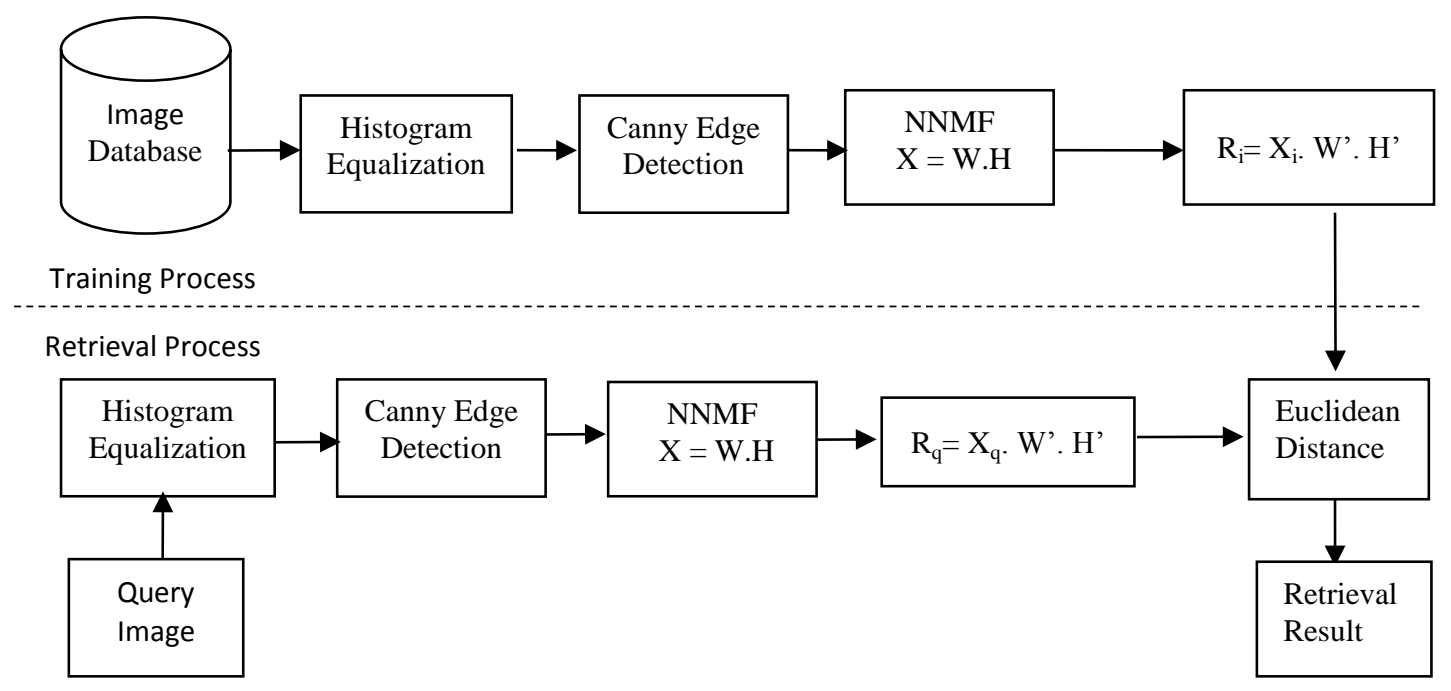

Figure 1. Medical Image Retrieval Process

Figure 1 shows that the processes which will be done are preprocessing by using histogram equalization, feature extraction by using canny edge detection, and features recognition by using non-negative matrix Factorization. All these processes will be applied to both training and retrieval process. Feature recognition resulted from training process will be stored into database. Then, feature recognition resulted from query image retrieval process will be compared with existing data in database. Euclidean distance method will be used to determine similarity between two images.

\section{Result and Discussion}

The tools used in this research is MATLAB 2012. The brain medical images will be divided into three classes, which are normal, alzheimer and glioma. Each classes consisted of 40 images. Each image will be resized into 256 x 256 pixels. Before the training or retrieval process of medical images. Preprocessing will be done by Histogram Equalization. It can make us easier when reading the information from medical image.

Tests that will be conducted consist of comparing the results of image retrieval by using a non-preprocessing image or preprocessing image. Based on the test that will be done, two types of data storage is needed one for non-preprocessing image and preprocessing image. The image sample that will be used in this research will be selected from each class. Each class provide ten images for query image. Images is chosen randomly from data source. These tests will result other ten most similar results compared with query image.

The tests process will begin by converting the query image into a grayscale image. After that, the feature extraction process with canny edge detection and recognition with Non-negative Matrix Factorization method will be done. The results of these NNMF will be compared with the results of NNMF existing in the database.

By using Matlab 2012, a snippet of program to make grayscaling, canny edge detection and non-negative matrix factorization process can be seen in Figure 2. 


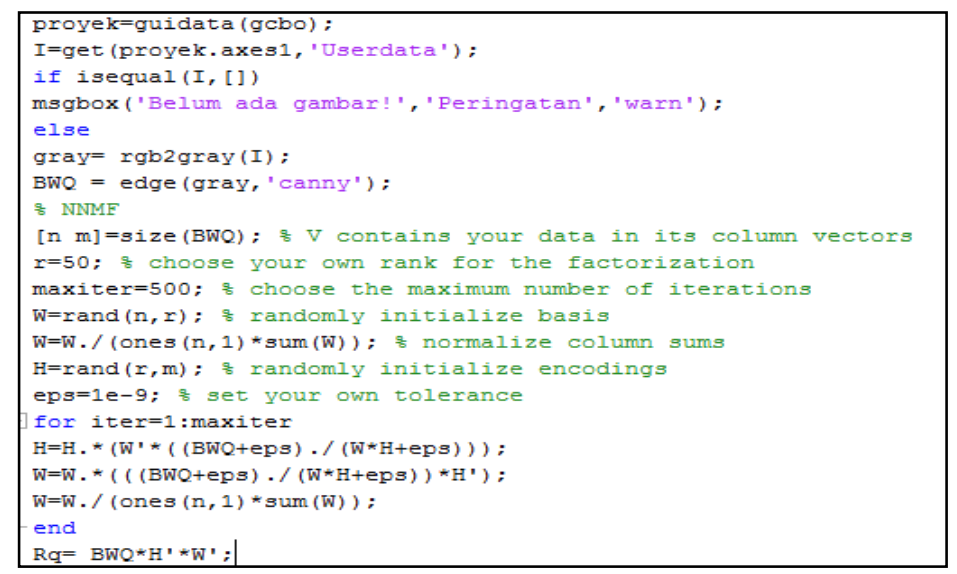

Figure 2. Non-preprocessing Snippet

Figure 2 shows that histogram equalization process is not will used in the preprocessing. So medical image will not changed and looks the same as image from the data source. Results of retrieval by using non-preprocessing image can be seen in Figure 3.

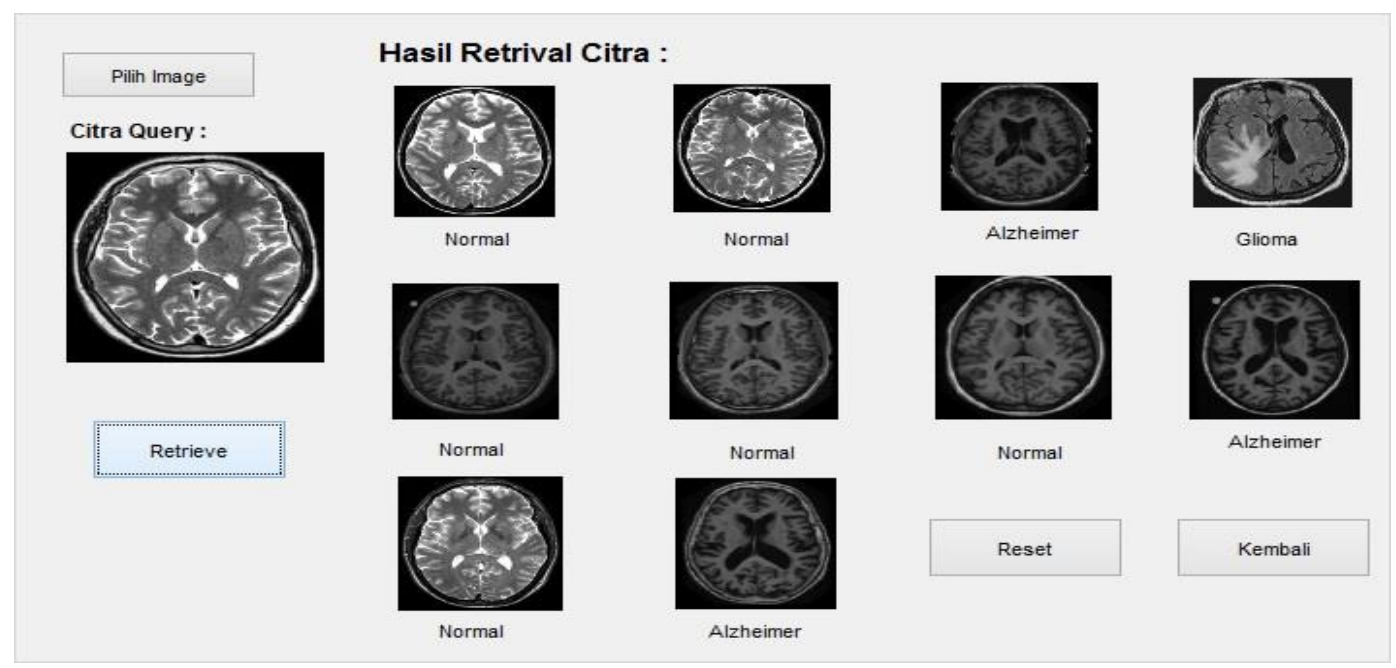

\section{Figure 3. The Image Retrieval Results with Query Image of Normal by Using Non-preprocessing Image}

Figure 3 shows that the retrieval system displayed the 10 most similar images with query image. Only six content image that relevant to the query image. After tests non-image preprocessing, the next testing is retrieval of preprocessing image. In this research, preprocessing by using histogram equalization.

In image retrieval process using image preprocessing, Histogram equalization is called from built-in function in Matlab 2012. For more details the use of histogram equalization function can be seen in the snippet in Figure 4. 


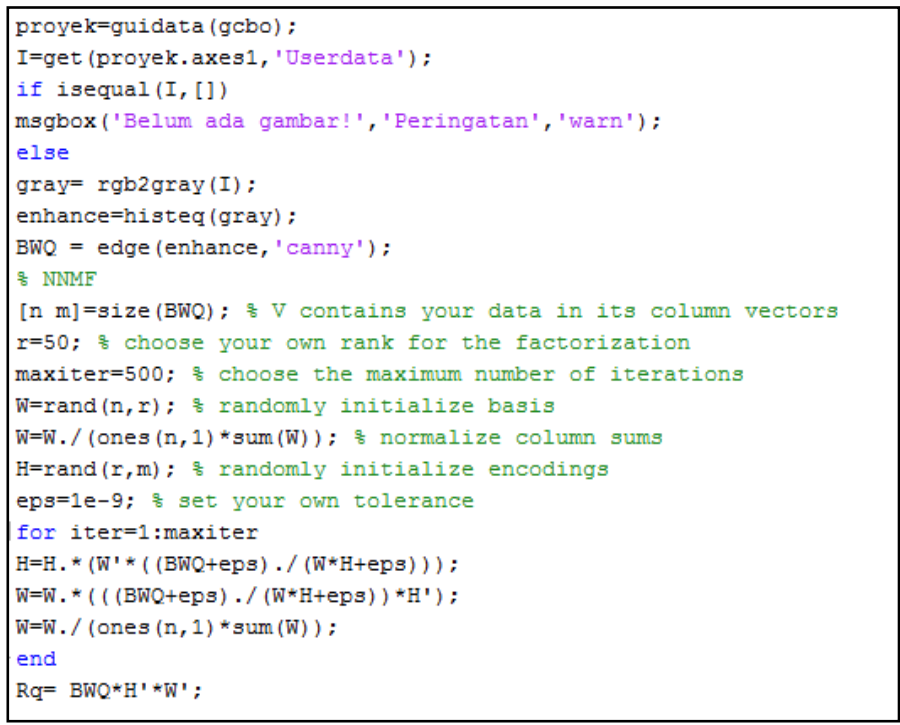

Figure 4. Preprocessing Program Snippet

Figure 4 shows that prior to the feature extraction with canny edge detection, image is processed with histogram equalization. Histogram equalization process aims to improve the quality of the features extraction. The results of preprocessing image retrieval with histogram equalization can be seen in Figure 5.

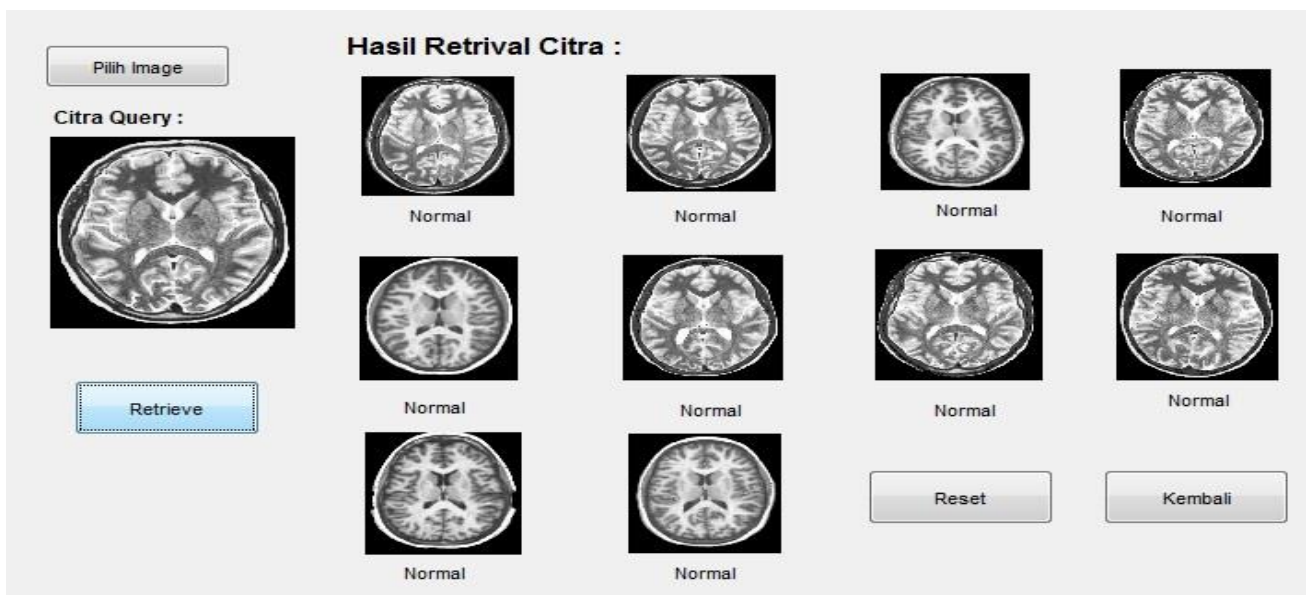

Figure 5. The Image Retrieval Results of with Query Image of Normal by Using Preprocessing Image

The results of image retrieval in Figure 5 shows that the retrieval system successfully retrieved 10 images that are relevant to the query image. There is difference between image retrieval result by using non-preprocessing image and preprocessing image. These different result are cause by the use of histogram equalization in preprocessing method.

Tests applied to 30 brain medical images consists of 10 normal brain medical images, 10 Alzheimer brain medical images and 10 glioma brain medical images. These tests will be applied with two different type which are 500 and 1000, and different type of k values are 10, 20, 50, and 100. These test are determine which method that produced a good average precision value. So the test results for each condition of the brain can be seen in Table 1. 
Table 1. Comparison Image Retrieval between Preprocessing Image and Non-preprocessing Image

\begin{tabular}{|c|c|c|c|}
\hline \multirow{2}{*}{ Condition } & \multirow{2}{*}{ rank } & \multicolumn{2}{|c|}{ Image } \\
\hline & & Preprocessing & Non-preprocessing \\
\hline \multirow{4}{*}{ Normal } & 10 & $92 \%$ & $55 \%$ \\
\hline & 20 & $83 \%$ & $47 \%$ \\
\hline & 50 & $77 \%$ & $47 \%$ \\
\hline & 100 & $68 \%$ & $39 \%$ \\
\hline \multicolumn{2}{|c|}{ Average Precision of Normal Brain } & $80 \%$ & $59 \%$ \\
\hline \multirow{4}{*}{ Glioma } & 10 & $61 \%$ & $65 \%$ \\
\hline & 20 & $57 \%$ & $66 \%$ \\
\hline & 50 & $58 \%$ & $71 \%$ \\
\hline & 100 & $61 \%$ & $75 \%$ \\
\hline \multicolumn{2}{|c|}{ Average Precision of Glioma Brain } & $60 \%$ & $66 \%$ \\
\hline \multirow{4}{*}{ Alzheimer } & 10 & $92 \%$ & $79 \%$ \\
\hline & 20 & $91 \%$ & $75 \%$ \\
\hline & 50 & $99 \%$ & $68 \%$ \\
\hline & 100 & $90 \%$ & $61 \%$ \\
\hline \multicolumn{2}{|c|}{ Average Precision of Alzheimer Brain } & $91 \%$ & $58 \%$ \\
\hline \multicolumn{2}{|c|}{ Average Precision of all } & $77 \%$ & $61 \%$ \\
\hline
\end{tabular}

Table 1 shows that the image retrieval process produced good precision value if using preprocessing image was used Histogram Equalization. Having in mind that the image retrieval process was better when image preprocessing was used, then further testing using only the image preprocessing. The results of a comparison average precision of each condition of the brain can be seen in Figure 6.

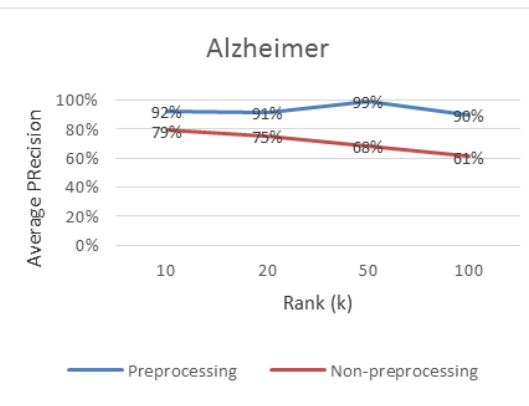

(a)

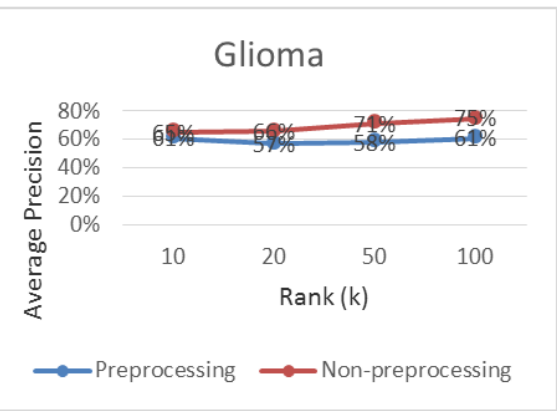

(b)

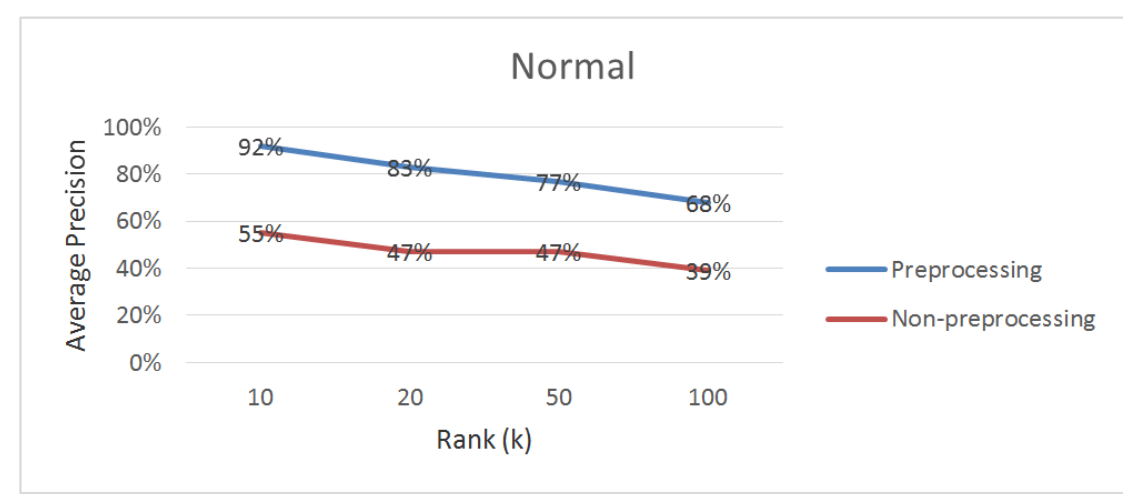

Figure 6. Average Precision of Image Retrieval; (a). Alzheimer Brain (b) Glioma Brain (c). Normal Brain 
By examining at the graph in Figure 6, it can be said that the use of image preprocessing could improve the retrieval process, especially for normal and Alzheimer brain condition. In conditions mentioned before the graphs showed a significant difference between preprocessing image and non-preprocessing image.

The next test was conducted to determine the maximum iteration value that produced the best precision value. As already known in earlier tests, the maximum iteration value are 500 and 1000 was used. The results of these tests can be seen in Table 2 .

\section{Table 2. Comparison of Average Precision with Different of Maximum Iteration Value}

\begin{tabular}{|c|c|c|}
\hline \multirow{2}{*}{ Condition } & \multicolumn{2}{|c|}{ Maximum iteration } \\
\cline { 2 - 3 } & 500 & 100 \\
\hline Normal & $80 \%$ & $81 \%$ \\
\hline Glioma & $59 \%$ & $61 \%$ \\
\hline Alzheimer & $93 \%$ & $81 \%$ \\
\hline Rata-rata & $\mathbf{7 7 \%}$ & $\mathbf{7 7 \%}$ \\
\hline
\end{tabular}

From Table 2, it can be concluded that the differences in the use of maximum iterations in this research did not affect the results of image retrieval. Therefore, to speed up the testing process, maximum iterations value of 500 was used. The next test was to determine factorization value or rank value $(\mathrm{k})$ which produces the best value of average precision. The test results can be seen in Table 3.

Table 3. Comparison of Average Precision with Different Rank Values

\begin{tabular}{|c|c|c|c|c|}
\hline \multirow{2}{*}{ Rank } & \multicolumn{3}{|c|}{ Condition } & \multirow{2}{*}{ Average } \\
\cline { 2 - 5 } & Normal & Alzheimer & Glioma & \\
\hline 10 & $92 \%$ & $92 \%$ & $61 \%$ & $\mathbf{8 2 \%}$ \\
\hline 20 & $83 \%$ & $91 \%$ & $57 \%$ & $\mathbf{7 7 \%}$ \\
\hline 50 & $77 \%$ & $99 \%$ & $58 \%$ & $\mathbf{7 8 \%}$ \\
\hline 100 & $68 \%$ & $90 \%$ & $61 \%$ & $\mathbf{7 3 \%}$ \\
\hline
\end{tabular}

Based on test results in Table 3, rank value (k) of 10 was provided better precision than rank value of 20,50, and 100. In addition, when the rank value of 10 was used processing time would be faster. Because the use of small rank value would produce a small matrix factorization. The test results can be seen in Figure 7. 


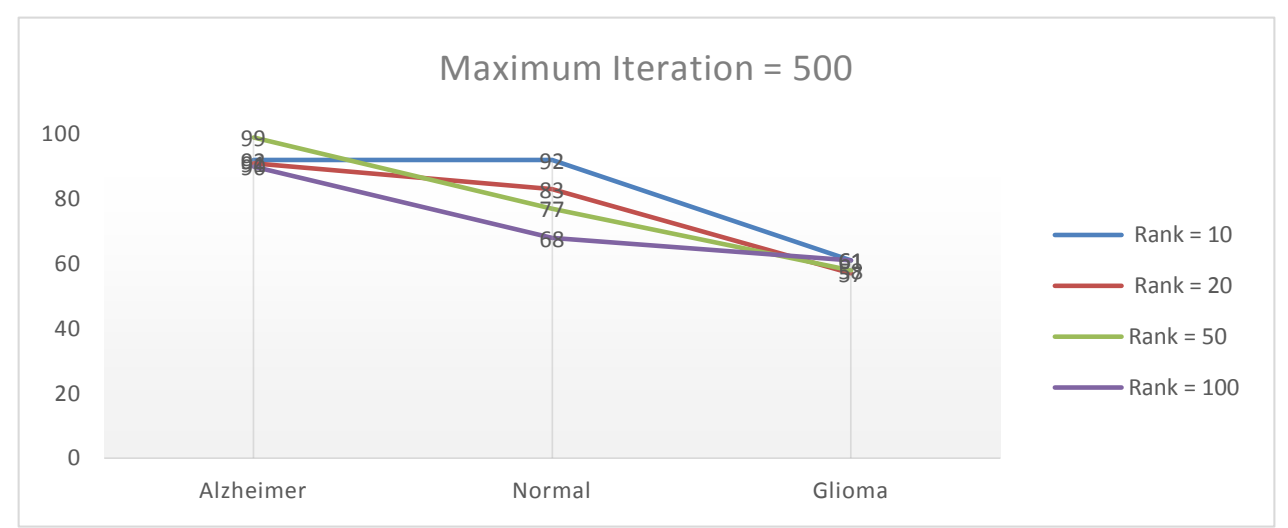

\section{Figure 7. Comparison of Average Precision with Maximum Iteration Value of 500 and the Different of Value Rank}

Figure 7 presents the comparison of average precision value when viewed from different of rank values. The results indicated that are using rank value of 10 would not make a significant decrease.

\section{Conclusion}

The average precision value of image retrieval using preprocessing with histogram equalization was better than without preprocessing. The value of average precision on preprocessing image was $77 \%$. And Maximum iterations value in this research did not affect average precision value in image retrieval process. In addition, rank value of 10 made better retrieval process than the rank value of 20,50 and 100. Average precision value at value rank of 10 was $82 \%$. And last, the retrieval process produced highest value of average precision in Alzheimer's and Normal image. Its average precision value was $92 \%$.

\section{References}

[1] A. G. Selvarani and S. Annadurai, "Content Based Medical Image Retrieval System using Multiple Classifier Framework", (2010), pp. 1-2.

[2] B. Ramamurthy, "Cbmir: Shape-Based Image Retrieval Using Canny Edge Detection And K-Means Clustering Algorithms For Medical Images", (2011), pp. 5-7.

[3] S. Rao, S. Kumar and C. Mohan, "Content Based Image Retrieval Using Exact Legendre Moments and Support Vector Machine", (2010), pp. 72-75.

[4] G. Quellec, M. Lamard, G. Cazuguel, B. Cochener and C. Roux, "Wavelet Optimization for Content Based Image Retrieval in Medical Databases", (2010), pp. 231-233.

[5] W. Liu and N. Zheng, "Learning Sparse Feature For Classification By Mixture Models", (2004), pp. 155-161.

[6] G. Buchsbaum and O. Bloch, "Color Categories Revealed By Non-Negative Matrix Factorization Of Munsell Color Spectra”, (2002).

[7] I. Buciu and I. Pitas, "Application Of Non-Negative And Local Non Negative Matrix Factorization To Facial Expression Recognition”, (2004), pp. 288-291.

[8] L. Gu, S. Li and H. Zhang, "Learning Probabilistic Distribution Model for Multimedia View Face Detection", (2001).

[9] I. Buciu, "Non-negative Matrix Factorization", A New Tool for Feature Extraction, vol. 1, (2008).

[10] M. W. Berry and J. Kogan, "Text Mining Application and Theory", (2010), pp. 70-72.

[11]C. Akgul, D. Rubin, F. Beaulieu, H. Greenspan and B. Acar, "Content-Based Image Retrieval in Radiology: Current Status and Future Directions”, (2011), pp. 208-222.

[12] J. F. Canny, “A Computational Approach to Edge Detection”, (1986), pp. 679-697.

[13] Raman, Maini, and Himanshu, "Aggarwal, Study and Comparison of Various Image Edge Detection Techniques", (2011), pp. 6-7.

[14] O. Okun, "Non-negative Matrix Factorization and Classifier : Experimental Study", (2004), pp. 550-555.

[15]D. D. Lee and H. S. Seung, “Algorithms for Non-negative Matrix Factorization”, (2000). 


\section{Authors}

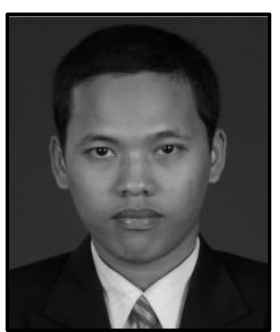

Ali Akbar Lubis, He is a lecturer at bachelor degree in STMIK Mikroskil. He received under graduated degree in information technology from Faculty of Information Technology, Mikroskil, Medan, Indonesia in 2010. He received master degree in information technology engineering from Bina Nusantara University, Jakarta, Indonesia, in 2014. His interest researches are computer science and image processing.

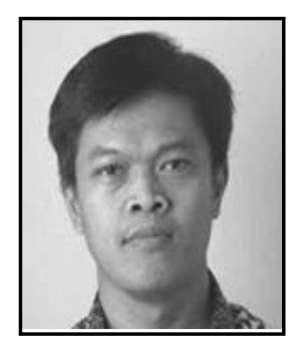

Suharjito, He is the Head of Information Technology Department in Binus Graduate Program of Bina Nusantara University. He received under graduated degree in mathematics from The Faculty of Mathematics and Natural Science, Gadjah Mada University, Yogyakarta, Indonesia in 1994. He received master degree in information technology engineering from Sepuluh November Institute of Technology, Surabaya, Indonesia in 2000. He received the $\mathrm{PhD}$ degree in system engineering from the Bogor Agricultural University (IPB), Bogor, Indonesia in 2011. His interest researches are intelligent system, Fuzzy system, image processing, image compression and software project estimation supply chain risk management. 
International Journal of Multimedia and Ubiquitous Engineering Vol.10, No.4 (2015) 